\title{
The Risk Factors of Coronary Heart Disease and its Relationship with Endothelial Nitric Oxide Synthase
}

\author{
Eka Fithra Elfi ${ }^{1 *}$, Eva Decroli ${ }^{2}$, Ellyza Nasrul ${ }^{3}$, Yanwirasti Yanwirasti ${ }^{4}$, Eryati Darwin ${ }^{5}$ \\ ${ }^{1}$ Department of Cardiology and Vascular Medicine, Faculty of Medicine, Universitas Andalas, Padang, Indonesia; ${ }^{2}$ Department \\ of Internal Medicine, Faculty of Medicine, Universitas Andalas, Dr. M. Djamil General Hospital, Padang; ${ }^{3}$ Department of Clinical \\ Pathology, Faculty of Medicine, Universitas Andalas, Padang, Indonesia; ${ }^{4}$ Department of Anatomy, Faculty of Medicine, Universitas \\ Andalas, Padang, Indonesia; ${ }^{5}$ Department of Histology and Immunology, Faculty of Medicine, Andalas University, Padang, Indonesia
}

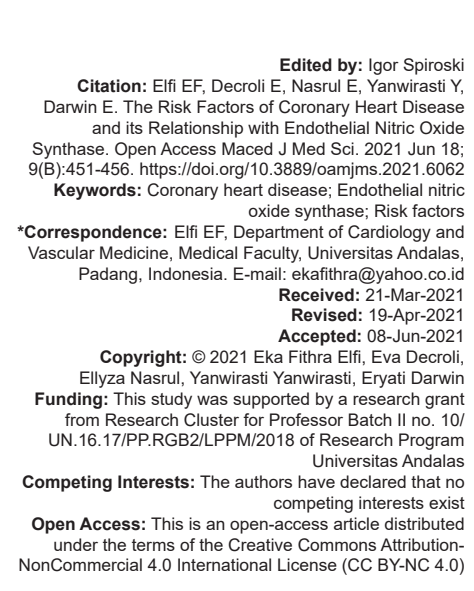

\begin{abstract}
BACKGROUND: Coronary heart disease (CHD) is the leading cause of death and start with injury to the endothelium of a coronary artery. The common feature of endothelial dysfunction is a decrease of nitric oxide (NO) bioavailability that regulated by endothelial NO synthase (eNOS) activity.

AIM: The aim of our study was to study the relationship between risk factors of CHD patients with the level of eNOS.

METHODS: Thirty-seven outpatients in cardiology department of the regional public hospital diagnosed as CHD were included in our study. Thirty healthy individuals were included as the control group. Risk factors of CHD were identified according to anamnesis and laboratory finding. eNOS was measured by ELISA methods.

RESULTS: Endothelial NOS levels were significantly higher in the CHD when compared to the controls $(p<0.05)$ The most dominant risk factor for CHD is overweight, and followed by dyslipidemia, smoking, hypertension, history of $\mathrm{CHD}$, and diabetes mellitus. eNOS in CHD patients who had one risk factor was $37.598 \pm 0.1541 \mathrm{ng} / \mathrm{ml}$, two risk factors $42.154 \pm 22.329 \mathrm{ng} / \mathrm{ml}$, three risk factors $25.329 \pm 6.083 \mathrm{ng} / \mathrm{ml}$, four risk factors $22.483 \pm 4.022 \mathrm{ng} / \mathrm{ml}$, and five risk factors $15.994 \pm 4.774 \mathrm{ng} / \mathrm{ml}$. There were significant differences in the average eNOS levels based on the number of risk factors $(p<0.05)$, and a tendency that more risk factors in CHD patients, the lower the average leve of eNOS.

CONCLUSION: In our study, eNOS levels showed highly significant relation with CHD and related to the number of risk factors those the $\mathrm{CHD}$ patients had.
\end{abstract}

\section{Introduction}

Cardiovascular diseases (CVDs) are a general term for diseases that affect the structures or function of the heart or blood vessels. There are a number of underlying determinants of CVDs include hereditary factors, live style, and poverty [1], [2]. Coronary heart disease (CHD), also called as ischemic heart disease or coronary artery disease, is a serious health problem worldwide, because it leads of the cardiovascular disability even death. In Indonesia, the incidence of CHD is increasing from year to year, so that the morbidity and mortality cause an economic and social burden on society [3], [4].

CHD develops as a result of injury or damage to the inner layer of coronary arteries, causes the narrowing or blockage of this vessels [5], [6], [7]. When a coronary artery is blocked by the clot long enough to cause irreversible damage, the heart muscle may die due to starved for nutrients and oxygen and this is called as myocardial infarction, commonly referred to as a heart attack [8], [9], [10], [11].
Chronic exposure of cardiovascular risk factors disrupts the defense mechanisms of the vascular endothelium, which is followed by endothelial dysfunction. Endothelial dysfunctions are usually characterized by the imbalance in the secretion and release of vasoconstriction and vasodilation mediators that affect the prothrombotic and proatherogenic activity of vascular endothelial [7]. Long-term impact of endothelial dysfunction leads to disruption of the vascular tone by imbalance of nitric oxide (NO) production and $\mathrm{NO}$ inactivation. Endothelial NO synthase (eNOS), the predominant NO synthase isoform in the vasculature, is responsible for most of the NO produced in this tissue. eNOS is a central regulator of cellular function that is important to maintain endothelial homeostasis. eNOS plays a role in coupling changes of blood flow to long-term remodeling of the vascular architecture [11], [12], [13].

The progression from endothelial dysfunction to atherosclerosis is related to deterioration of endothelial function, impairment of vascular homeostasis that leads to reduced antioxidant and anti-inflammatory effects, increased vascular permeability, and the increased 
expression of inflammatory cytokines and adhesion molecules [14]. Oxidative stress appears to be the most common underlying mechanism for the development of endothelial dysfunction. Most cardiovascular risk factors are associated with the upregulation of intracellular oxidative stress, reactive oxygen species (ROS), and $\mathrm{NO}$ inactivation. $\mathrm{NO}$ is produced and released from L-arginine through the activity of the eNOS. In the healthy endothelium, eNOS is responsible for most of the vascular NO production, while in pathologic or unhealthy endothelium, eNOS becomes a potential ROS generator [15], [16].

To analyze the risk factors of CHD and its relation to the one of endothelial dysfunction marker, we studied the relationships between modifiable and non-modifiable risk factors of CHD with the level of eNOS in CHD patients and healthy persons.

\section{Methods}

\section{Subject}

This study was a cross-sectional study; conducted in 37 patients of the outpatients in department cardiology of regional public hospital, which is carried out within a period of 11 months. Inclusion criteria were patients in the age between 30 and 60 years, diagnosed as CHD by the cardiologist clinically with electrocardiogram (ECG) and laboratory confirmation and who agree to participate in this study. Exclusion criteria of the study were defined as the presence of infectious diseases, and a history of malignancies and other autoimmune diseases. Thirty healthy individual who come to the department cardiology for medical examinations to obtain a medical certificate, which evaluated using ECG and had no history of risk factors for CHD were identified as the control group and responded to the questionnaire. This study had obtained informed consent from all subjects and was approved by Research Ethic Committee of Faculty of Medicine Universitas Andalas.

\section{Risk factors}

The non-modifiable risk factors for CHD, such as gender, age, and family history of CHD were assessed using questioner with several questions. The modifiable risk factors including dyslipidemia, hypertension, diabetes mellitus (DM), overweight, and smoking were determined by history, physical, and laboratory examinations. In this study, subjects were grouped into groups that had one to six risk factors, including dyslipidemia, hypertension, DM, overweight, smoking, and family history of CHD.
Dyslipidemia was confirmed when the levels of triglyceride were $>150 \mathrm{mg} / \mathrm{dl}$, low-density lipoprotein (LDL) greater $100 \mathrm{mg} / \mathrm{dl}$, high-density lipoprotein (HDL) $<50 \mathrm{mg} / \mathrm{dl}$, and total cholesterol $>200 \mathrm{mg} / \mathrm{dl}$. Hypertension was determined if blood pressure was higher than $140 / 90 \mathrm{mmHg}$. DM was determined by the level of fasting blood glucose more than $126 \mathrm{mg} / \mathrm{dl}$. Height and body weight were measured to calculate body mass index (BMI) for determining overweight (BMI in range 25-29.9 kg/m²) or obese (BMI more than $30 \mathrm{~kg} / \mathrm{m}^{2}$ ). An active smoker was defined as a person who regularly smokes at least one cigarette a day in past 1 year. Family history was obtained by asking about CHD history occurring in the first-degree relatives (mother, father, and siblings) and second-degree relatives (aunts, uncles, and grandparents). All methods were carried out in accordance with relevant guidelines and regulations [4], [11], [17].

\section{Measurement of eNOS}

Fasting blood samples were obtained by direct venipuncture and drawn into vacutainer tubes at room temperature containing tripotassium ethylenediaminetetraacetic acid (K3-EDTA) from the antecubital veins in all subjects. Serum eNOS was determined using a commercially available sandwich enzyme immunoassay kit and were calculated using enzyme-linked immunosorbent assay (ELISA) methods. Human eNOS ELISA kit from Bioassay Technology Laboratory Shanghai, China (Abcam E0908 Hu) is an in vitro ELISA for the measurement of human pan-eNOS and total eNOS. The eNOS antibody has been coated onto a 96-well plate. Blood samples were centrifuged for $20 \mathrm{~min}$ at $\times 1000 \mathrm{~g}$ at $4{ }^{\circ} \mathrm{C}$, and then, $40 \mu \mathrm{l}$ serum of blood samples were pipetted into the wells and eNOS present in a sample is bound to the wells by the immobilized antibody. The wells were washed and $10 \mu \mathrm{l}$ mouse anti-eNOS antibody is used to detect eNOS. After washing away unbound antibody, $50 \mu \mathrm{l}$ HRP-conjugated anti-mouse IgG is pipetted to the wells. The wells were again washed, a $90 \mu \mathrm{l}$ TMB substrate solution was added to the wells and color develops in proportion to the amount of eNOS or eNOS bound. The stop solution changes the color from blue to yellow, and the intensity of the color is measured at $450 \mathrm{~nm}$. All of laboratory examinations are carried out according to the manufacturer and laboratory standards in Biomedical Laboratory of Medical Faculty Universitas Andalas [17].

\section{Ethical approval}

This study was conducted according to the guidelines of the Declaration of Helsinki 1975, as revised in 2000 . The procedure was approved by the 
Ethics Research Committee of Faculty of Medicine Universitas Andalas. All participants who agreed to participate in the study by signing the personal informed consent.

\section{Statistical analysis}

Quantitative data obtained in the study were expressed as the mean \pm standard deviation, whereas qualitative values are given in percentages. Comparisons between the groups were carried out using ANOVA and post hoc Bonferroni test as appropriate. $p<0.05$ was considered to indicate a statistically significant difference.

\section{Results}

The present study involves $37 \mathrm{CHD}$ patients consisting of $64.86 \%$ men and $35.14 \%$ women in the age range of 34-55 years old and 30 healthy persons as the control, consisting of $66.67 \%$ men and $33.33 \%$ women in the age range of 35-55 years old. On this study, from the risk factor of CHD that can be controlled include dyslipidemia, hypertension, DM, overweight, and smoking habits, added with one risk factor that cannot be controlled, namely, history of family with $\mathrm{CHD}$, is six types of risk factor to be analyzed. The most dominant risk factor from six risk factors of CHD is overweight which is experienced by $72.97 \%$ of $\mathrm{CHD}$ patients, followed by dyslipidemia on $64.86 \%$ of $\mathrm{CHD}$ patients, smoking habits on $56.76 \%$ of CHD patients, hypertension on $54.05 \%$ of CHD patients, family history of CHD on $32.43 \%$ of CHD patients, and DM on $24.32 \%$ of CHD patients.

CHD patients of the present study have one to six risk factors of $\mathrm{CHD}$ as seen in Table 1, where $5.4 \%$ of CHD patients have one risk factor from six risk factors, $21.6 \%$ of $\mathrm{CHD}$ patients have two risk factors, $43.3 \%$ of $\mathrm{CHD}$ patients have three from 6 risk factors, $18.9 \%$ have four from six risk factors of $\mathrm{CHD}$, and $10.6 \%$ of CHD patients have five from six risk factors of CHD. There are no patients having the predetermined six risk factors in this study.

It is seen that the eNOS levels on CHD patients with one risk factor are $37.598 \pm 0.1541(\mathrm{ng} / \mathrm{ml})$, two risk factors $42.154 \pm 22.3290(\mathrm{ng} / \mathrm{ml})$, three risk factors $25.328 \pm 6.0835(\mathrm{ng} / \mathrm{ml})$, four risk factors $22.483 \pm 4.0224$ (ng/ml), and five risk factors $15.994 \pm 4.7739(\mathrm{ng} / \mathrm{ml})$. By utilizing ANOVA, there are significant differences of the mean of eNOS levels based on the number of risk factor $(p=0.003)$. With the post hoc test of Benferroni it is seen that the level of eNOS on CHD patients having one risk factor is not significantly different in statistic with the level of eNOS on CHD patients having two more than two CHD risk factors $(p=0.372)$. The levels of eNOS
Table 1: Types and number of risk factor on coronary heart disease (CHD) patients

\begin{tabular}{|c|c|c|c|c|c|c|}
\hline \multirow{2}{*}{$\begin{array}{l}\text { Number of } \\
\text { Risk Factor }\end{array}$} & \multirow[t]{2}{*}{ Type of risk factor } & \multirow[t]{2}{*}{$\mathrm{n}$} & \multicolumn{2}{|l|}{ Total } & \multirow{2}{*}{$\begin{array}{l}\text { eNOS } \\
\text { Mean } \pm \text { SD }\end{array}$} & \multirow[t]{2}{*}{$\mathrm{p}$} \\
\hline & & & Amount & $\%$ & & \\
\hline 1 risk factor & Dyslipidemia & 2 & 2 & 5.4 & $37.598 \pm 0.1541$ & 0.003 \\
\hline \multirow[t]{6}{*}{2 risk factors } & Smoking, dyslipidemia & 2 & 8 & 21.6 & $42.154 \pm 22.329$ & \\
\hline & Smoking, family history of & 1 & & & & \\
\hline & $\begin{array}{l}\text { CHD } \\
\text { Dyslipidemia, family history } \\
\text { of } \mathrm{CHD}\end{array}$ & 1 & & & & \\
\hline & Hypertension, overweight & 2 & & & & \\
\hline & Dyslipidemia, overweight & 1 & & & & \\
\hline & $\begin{array}{l}\text { Overweight, family history } \\
\text { of CHD }\end{array}$ & 1 & & & & \\
\hline \multirow[t]{8}{*}{3 risk factors } & $\begin{array}{l}\text { Dyslipidemia, smoking, CHD } \\
\text { history }\end{array}$ & 2 & 16 & 43.3 & $25.328 \pm 6.0835$ & \\
\hline & $\begin{array}{l}\text { Dyslipidemia, hypertension, } \\
\text { CHD family history }\end{array}$ & 1 & & & & \\
\hline & $\begin{array}{l}\text { Dyslipidemia, DM, family } \\
\text { history of CHD }\end{array}$ & 1 & & & & \\
\hline & $\begin{array}{l}\text { Hypertension, overweight, } \\
\text { smoking, }\end{array}$ & 5 & & & & \\
\hline & $\begin{array}{l}\text { Dyslipidemia, overweight, } \\
\text { smoking }\end{array}$ & 3 & & & & \\
\hline & $\begin{array}{l}\text { Dyslipidemia, hypertension, } \\
\text { overweight }\end{array}$ & 2 & & & & \\
\hline & Dyslipidemia, DM, overweight & 1 & & & & \\
\hline & $\begin{array}{l}\text { Dyslipidemia, hypertension, } \\
\text { DM, family history of CHD }\end{array}$ & 1 & & & & \\
\hline \multirow[t]{6}{*}{4 risk factors } & $\begin{array}{l}\text { Dyslipidemia, hypertension, } \\
\text { overweight, smoking }\end{array}$ & 1 & 7 & 18.9 & $22.483 \pm 4.022$ & \\
\hline & $\begin{array}{l}\text { Dyslipidemia, DM, } \\
\text { overweight, smoking }\end{array}$ & 2 & & & & \\
\hline & $\begin{array}{l}\text { Dyslipidemia, hypertension, } \\
\text { DM, overweight }\end{array}$ & 2 & & & & \\
\hline & $\begin{array}{l}\text { Dyslipidemia, overweight, } \\
\text { smoking, family history of } \\
\text { CHD }\end{array}$ & 1 & & & & \\
\hline & $\begin{array}{l}\text { Dyslipidemia, hypertension, } \\
\text { overweight, family history } \\
\text { of CHD }\end{array}$ & 1 & & & & \\
\hline & $\begin{array}{l}\text { Dyslipidemia, Hypertension, } \\
\text { DM, overweight, smoking }\end{array}$ & 2 & & & & \\
\hline \multirow[t]{2}{*}{5 risk factors } & $\begin{array}{l}\text { Dyslipidemia, Hypertension, } \\
\text { DM, overweight, smoking }\end{array}$ & 2 & 4 & 10.8 & $15.994 \pm 4.773$ & \\
\hline & $\begin{array}{l}\text { Dyslipidemia, hypertension, } \\
\text { overweight, smoking, family } \\
\text { history of CHD }\end{array}$ & 2 & & & & \\
\hline Total & & 37 & 100 & & $28.082 \pm 13.845$ & \\
\hline
\end{tabular}

are higher on $\mathrm{CHD}$ patients having two $\mathrm{CHD}$ risk factors compared to CHD patients having three, four, and five CHD risk factors $(p<0.05)$, and it seems the tendency that the higher risk factors on $\mathrm{CHD}$ patients, the lower eNOS levels are.

From the examination of eNOS serum levels, it is seen that the mean of eNOS level on CHD patients is $28.082 \pm 13.846$, lower than the control group (71.316 $\pm 31.044)$ with the difference which is significant statistically $(p<0.001)$ (Table 2$)$.

Table 2: The difference between the mean of eNOS $(\mathrm{ng} / \mathrm{ml})$ levels on CHD patients with control group

\begin{tabular}{llll}
\hline Group & $\mathrm{n}$ & $\begin{array}{l}\text { eNOS } \\
(\text { mean } \pm \text { SD) }\end{array}$ & $\mathrm{p}$ \\
\hline CHD patients & 37 & $28.082 \pm 13.846$ & $<0.001$ \\
Control & 30 & $71.316 \pm 31.044$ & \\
\hline
\end{tabular}

CHD: Coronary heart disease, eNOS: Endothelial nitric oxide synthase,

\section{Discussion}

CHD is more common in men than in women. Men are 3-5 times more likely to have $\mathrm{CHD}$ than 
women. The incidence of CHD rapidly increases with age and that women have rates that are similar to those of men 10 years younger [18]. In this study, it was found that CHD in men were higher than woman in the age between 35 and 60 years old. This is related to levels of sex hormones in women and men. For women, the effects of menopause, including the loss of the hormone estrogen, appear to increase the risk of CHD. Estrogens might enhance vascular tonus in the microcirculation of woman, include vasodilatation, increasing the synthesis and bioactivity of $\mathrm{NO}$, regulating the production of prostaglandins, and expression of eNOS. Increasing age in men and woman reduces the ability of vascular vasodilatations, due to an increased production of oxygen-derived free radicals reducing the bioavailability of $\mathrm{NO}$, and reduced expression of eNOS [19], [20]. Study of Smith et al., 2006, suggests a mechanism that explains the persistent loss of eNOS activity and endothelial-derived NO availability in aging conduit arteries [21].

From this study, overweight as a controllable risk factor was found in $72.97 \%$ of $\mathrm{CHD}$ patients. The prevalence of overweight and obesity has increased over the past two decades in Indonesian. The prevalence is higher in boys than girls among children, but higher in females in the adolescent and adult. The prevalence of overweight and obesity is also higher in those living in urban areas and with the higher income or education [4]. Excess body fat is more prone to develop heart disease even if they have no other risk factors. Excess weight increases the strain on the heart, raises blood pressure, blood cholesterol and triglyceride levels, and lowers HDL. It is also associated with the development of DM. The pathogenesis of $\mathrm{CHD}$ in obesity is not well understood but probably relates to the effect of the metabolic syndrome and inflammatory responses due to the increasing of adipose tissue [22]. Changes in lifestyle such as physical activities coupled with diet-induced weight loss have the potential and were identified to reduce the inflammatory biochemical markers level associated with vascular endothelial dysfunction in obesity [16].

Dyslipidemia and DM found in $64.86 \%$ and $54.05 \%$, respectively, of the CHD patients in this study. Hypercholesterolemia, high levels of total cholesterol and LDL cholesterol result in impaired endothelial function in both peripheral and coronary circulation. Hyperglycemia in diabetic state may lead to increase oxidative stress and oxidized lipoproteins, especially small dense LDL. The high levels of fatty acids and hyperglycemia have also both been shown to induce an increased level of oxidation of phospholipids as well as proteins; it is associated with a prothrombotic tendency as well as increased platelet aggregation [15].

From the data of this study, active smokers were found in $56.76 \%$ of patients with CHD. It is well known that an active or passive smoker leads to accelerated development of CHD. Chronic exposure to cigarette smoke is associated with impairment of endotheliumdependent vasodilation. This appears to be due to an action of nicotine causing an increase of asymmetric dimethylarginine and production of oxygen-derived free radicals, resulting in a lower availability of NO [13]. These harmful effects of cigarette smoke were related to uncoupling of eNOS due to decreased availability of tetrahydrobiopterin as an important cofactor of eNOS. Several studies are defined that cigarette smoking acutely decreases chemotaxis by blockade of vascular endothelial growth factor-stimulated $\mathrm{NO}$, indicating a significant endothelial activation and injury with functional impairment of the vascular endothelium. Taken together, cigarette smoking appears to not only acutely injure the vascular endothelium and block eNOS activity but also interfere with the vascular regenerative capacity [23], [24].

In our study, hypertension was found in $54.05 \%$ of CHD patients. Hypertension as a major risk factor for heart disease will increase the strain on arteries and can convert mechanical stimuli into intracellular or biochemical signals of vascular wall. Hypertension is related to several factors including free radical and inflammatory mediator's formation, impaired capillary blood flow, and inactivation of NO. The interaction of these factors will cause endothelial dysfunction associated with atherosclerosis [25].

The etiology for CHD is multifactorial, involving genetic and environmental components. Vinkhuyzen et al. [26] estimated that on the basis of family and twin studies, the heritability of CHD has been estimated between $40 \%$ and $60 \%$. Common variant association studies have confirmed about 60 genetic loci with a robust association with coronary disease. Genetic risk and combined with lifestyle factors and behaviors have a log-additive effect on the risk of developing CAD. Family history of CHD is associated with enhanced development and progression disease [27]. In the present study, family history of CHD is associated on $32.43 \%$ of CHD patients. The limitations of our study include the potential for recall bias of family history, and the use of different categorical variable of family history.

The various risk factors of $\mathrm{CHD}$ may be as physical or biochemical stimulus that trigger an immune response and lead to damage endothelial cells (Table 3). Endothelial dysfunction could be due to decreased eNOS expression. In this study, we found that eNOS levels on CHD patients were lower than the control group. eNOS that expressed by endothelium is a central regulator of endothelial function that is important to maintain vascular homeostasis. eNOS that generates NO plays a crucial role to maintain vascular, integrity due to its anti-inflammatory, antiproliferative, and antithrombogenic effects [13]. The level of eNOS is seen to be lower in relation to the increase in the number of risk factors that $\mathrm{CHD}$ patients have. Sequentially, the number of risk factors for $\mathrm{CHD}$ patients is overweight, dyslipidemia, smoking habits, hypertension, family history of $\mathrm{CHD}$, and $\mathrm{DM}$, which are related to the 
Table 3: The difference of the mean of eNOS $(\mathrm{ng} / \mathrm{ml})$ levels on $\mathrm{CHD}$ patients based on the number of CHD risk factor

\begin{tabular}{llll}
\hline Number of CHD risk factor & $\mathrm{n}$ & $\begin{array}{l}\text { eNOS } \\
\text { Mean } \pm \text { SD }\end{array}$ & $\mathrm{p}$ \\
\hline 1 risk factor & 2 & $37.598 \pm 0.1541$ & 0.003 \\
2 risk factors & 8 & $42.154 \pm 22.329$ & \\
3 risk factors & 16 & $25.328 \pm 6.0835$ & \\
4 risk factors & 7 & $22.483 \pm 4.022$ & \\
5 risk factors & 4 & $15.994 \pm 4.773$ & \\
Total & 37 & $28.082 \pm 13.845$ & \\
\hline CHD: Coronary heart disease, eNOS: Endothelial nitric oxide synthase.
\end{tabular}

severity of injury that occurs in the endothelium. These pathological stimuli contribute to excessive generation of ROS, therefore causing the imbalance that leads to cell and tissue damage. Moreover, tissue damage or oxidative stress could induce vascular inflammation through activation of cytokine and recruitment of inflammatory cells [28], [29]. eNOS is primarily responsible for the generation of NO in the vascular endothelium. Deficient of eNOS becomes the reduced activation and bioavailability and of NO produced from eNOS. Such inactivation leads to the loss of NO protective effects such as arterial tonus regulation, inhibition of local inflammation and coagulation, and cellular proliferation. Experimental studies in vitro have demonstrated that a deficiency of NO from eNOS constitutes as an anti-atherogenic molecule was related to accelerate atherosclerotic formation in the coronary arteries, that is, responsible for $\mathrm{CHD}$ [9], [30].

Endothelial dysfunction is a hallmark of most CVDs risk factors. Oxidative stress and inflammation represent common key pathways associated with these risk factors and diseases that trigger the adverse redox regulation of eNOS function [7]. A recent study proposed future therapeutic strategies that could be used to target eNOS activity and function in CVDs, including lifestyle changes. Several antioxidants or micronutrient such as folic acid, zinc, Vitamin C, and Vitamin E have proved effective in improvement of endothelial dysfunction in animal models and in patients with cardiovascular risk factors through stabilizing effects and recouple of eNOS, thereby increasing NO production. eNOS can be an important target for prevention of atherosclerosis following traditional lipid-lowering, anti-platelet, and vasodilator drugs [10], [31], [32]. Although endothelial dysfunction in CHD risk factors could be related to decreased eNOS expression, several studies have shown that cardiovascular risk factors are associated with an increase rather than a decrease in eNOS expression. It is likely to be a consequence of an excess production of $\mathrm{H}_{2} \mathrm{O}_{2}$ that increase eNOS expression through transcriptional and post-transcriptional mechanisms [18]. Besedina, 2016, showed that the eNOS activities in middle-aged and olderaged patients with $\mathrm{CHD}$ associated with $\mathrm{HT}$ lower than in healthy individuals of the same age group. Alterations in eNOS activity are more expressed in patients with CHD associated with HT [33] so that the study of eNOS in cardiovascular diseases is still needed.

\section{Conclusion}

Risk factors of $\mathrm{CHD}$ are exo- and endo-genous harmful factors that can cause endothelial dysfunction through interaction with injurious effects of oxidative stress. Expression and activity of eNOS are intimately involved in these processes. The association of a number of risk factors with the level of eNOS increased the risk of the development of atherosclerosis in CHD. The role of eNOS in maintaining vascular functional integrity appears to be important in preventing CHD in the future.

\section{References}

1. World Health Organization. Global health risks: mortality and burden of disease attributable to selected major risks. Geneva, Switzerland: World Health Organization; 2009.

2. World Health Organization. Cardiovascular diseases (CVDs). Geneva, Switzerland: World Health Organization; 2017.

3. Ferreira-González I. The epidemiology of coronary heart disease. Rev Esp Cardiol. 2014; 67: 139-144. https://doi. org/10.1016/j.rec.2013.10.002

4. Siswanto. Basic Health Research 2018. Directorate of NonCommunicable Disease Prevention and Control, Research and Development Council. Ministry of Health of the Republic of Indonesia. 2019.

5. Ganz P, Hsue PY. Endothelial dysfunction in coronary heart disease is more than a systemic process. Eur Heart J. 2013; 34(27): 2025-7.

6. Gutiérrez E, Flammer AJ, Lerman LO, Elízaga J, Lerman A, Fernández-Avilés F. Endothelial dysfunction over the course of coronary artery disease. Eur Heart J. 2013; 34(41): 3175-81.

7. Widmer RJ, Lerman A. Endothelial dysfunction and cardiovascular disease. Glob Cardiol Sci Pract. 2014; 2014(3): 291-308.

8. Sayols-Baixeras S, Lluís-Ganella C, Lucas G, Elosua R. Pathogenesis of coronary artery disease: focus on genetic risk factors and identification of genetic variants. Appl Clin Genet. 2014; 7 : 15-32.

9. Ambrose JA, Singh M. Pathophysiology of coronary artery disease leading to acute coronary syndromes. F1000Prime Rep. 2015; 7: 08.

10. Bergheanu SC, Bodde MC, Jukema JW. Pathophysiology and treatment of atherosclerosis: Current view and future perspective on lipoprotein modification treatment. Neth Heart $\mathrm{J}$. 2017; 25(4): 231-42.

11. Hajar R. Risk factors for coronary artery disease: historical perspectives. Heart Views. 2017; 18(3): 109-14.

12. Förstermann $U$, Sessa WC. Nitric oxide synthases: regulation and function. Eur Heart J. 2012; 33(7): 829-37.

13. Heiss C, Rodriguez-Mateos A, Kelm M. Central role of eNOS in the maintenance of endothelial homeostasis. Antioxid Redox Signal. 2015; 22(14): 1230-42.

14. Park KH, Park WJ. Endothelial dysfunction: clinical implications in cardiovascular disease and therapeutic approaches. J Korean Med Sci. 2015; 30(9): 1213-25.

15. Hadi AR, Cornelia S, Carr CS, Al Suwaidi J. Endothelial dysfunction: cardiovascular risk factors, therapy, and outcome. Vasc Health Risk Manag. 2005; 1(3): 183-98. 
16. Kwaifa IK, Bahari H, Yong YK, Md Noor S. Endothelial dysfunction in obesity-induced inflammation: molecular mechanisms and clinical implications. Biomolecules. 2020;10(2): 291.

17. Ali, H. Standard operating procedures for ELISA of Biomedical Laboratory of Medical Faculty Universitas Andalas, Padang Indonesia, 2019

18. Zhang Y. Cardiovascular Diseases in American Women. Nutr Metab Cardiovasc Dis. 2010; 20(6): 386-393.

19. Förstermann U, Münzel T. Endothelial nitric oxide synthase in vascular disease. From Marvel to Menace. Circulation. 2006; 113: $1708-14$.

20. Maas AHEM, Appelman YEA. Gender differences in coronary heart disease. Neth Heart J. 2010; 18(12): 598-602.

21. Smith AR, Visioli F, Frei B, and Hagen TM. Age-related changes in endothelial nitric oxide synthase phosphorylation and nitric oxide dependent vasodilation: evidence for a novel mechanism involving sphingomyelinase and ceramide-activated phosphatase. Aging Cell, 2006; 5:391-400

22. Fuster JJ, Ouchi N, Gokce N,1 and Walsh K. Obesity-induced Changes in Adipose Tissue Microenvironment and Their Impact on Cardiovascular Disease. Circ Res., 2016; 118(11): 1786-1807.

23. Wagner L, Laczy B, Tamaskó M, Mazák M, Markó L, Molnár LA, et al. Cigarette smoke-induced alterations in endothelial nitric oxide synthase phosphorylation: role of protein kinase C. Endothelium. 2007; 14(4-5): 245-55.

24. World Tobacco Free Day. Center for Data and Information, Ministry of Health of the Republic of Indonesia. Info Datin 2014.

25. Konukoglu D, Uzun H. Endothelial dysfunction and hypertension.
Adv Exp Med Biol. 2016: 1-31.

26. Vinkhuyzen AA, Wray NR, Yang J, Goddard ME, Visscher PM. Estimation and partition of heritability in human populations using whole-genome analysis methods. Annu Rev Genet. 2013; 47: 75-95.

27. Said MA, van de Vegte YJ, Zafar MM, van der Ende MY, Raja GK, Verweij N, van der PHarst. Contributions of interactions between lifestyle and genetics on coronary artery disease risk. Curr Cardiol Rep. 2019; 21(89): 1-8.

28. Förstermann U, Xia N, Li H. Roles of vascular oxidative stress and nitric oxide in the pathogenesis of atherosclerosis. Circ Res. 2017; 120: 713-35.

29. Pizzino G, Irrera N, Cucinotta M, Pallio G, Mannino F, Arcoraci V, et al. Oxidative stress: harms and benefits for human health. Oxid Med Cell Longev. 2017; 2017; 1-13.

30. Kawashima $S$ and Yokoyama M. Dysfunction of endothelial nitric oxide synthase and atherosclerosis. Arterioscler Thromb Vasc Biol. 2004; 24(6): 998-1005.

31. Hong FF, Liang XY, Liu W, Sha LV, He SJ, Kuang HB, et al. IRoles of eNOS in atherosclerosis treatment. Inflamm Res. 2019; 68: 429-441. https://doi.org/10.1007/s00011-019-01229-9

32. Daiber A, Xia N, Steven S, Oelze M, Hanf A, Kröller-Schön S,1 Münzel, Li H. New therapeutic implications of endothelial nitric oxide synthase (eNOS) function/dysfunction in cardiovascular disease. Int J Mol Sci. 2019; 20(1): 187.

33. Besedina A. NO-Synthase Activity in Patients with Coronary Heart Disease Associated with Hypertension of Different Age Groups. J Med Biochem., 2016; 35(1): 43-49. 